\title{
Reducing HIV risk among adolescent girls and young women, and their partners through the DREAMS Partnership in Malawi
}

Project SOAR

Follow this and additional works at: https://knowledgecommons.popcouncil.org/departments_sbsr-hiv

Part of the Demography, Population, and Ecology Commons, Family, Life Course, and Society Commons, International Public Health Commons, Medicine and Health Commons, and the Women's Health Commons How does access to this work benefit you? Let us know!

\section{Recommended Citation}

Project SOAR. 2017. "Reducing HIV risk among adolescent girls and young women, and their partners through the DREAMS Partnership in Malawi," Activity brief. Washington, DC: Population Council. 


\section{Reducing HIV Risk among Adolescent Girls and Young Women, and Their Partners Through the DREAMS Partnership in Malawi}

Over the last decade, Malawi has witnessed a dramatic reduction in the annual number of new HIV infections among adults aged 15-64 from 98,000 in 2005 to 28,000 in $2016 .{ }^{1}$ Despite this impressive progress, the severity of the epidemic continues to vary considerably across sub-populations and geography within the country. Adolescent girls and young women (AGYW) in Malawi, like in many African countries, experience much higher rates of HIV infection than their male counterparts. Those living in urban areas are three times more likely to be HIV positive than their rural peers. Social isolation, economic disadvantage, discriminatory cultural norms, gender-based violence, school dropout, child marriage, and biological factors all contribute to AGYW's vulnerability to HIV.

The DREAMS (Determined, Resilient, Empowered AIDS-free, Mentored, and Safe) partnership aims to significantly reduce new HIV infections in AGYW in 10 countries in sub-Saharan Africa, including Malawi. DREAMS is delivering, through local and international implementing partners, a comprehensive package of evidence-based

Research Partners: College of Medicine at the University of Malawi

Location: Machinga and Zomba Districts

Study Duration: 2015-2018

For more information, Nanlesta Pilgrim (npilgrim@popcouncil. org), Sanyukta Mathur (smathur@ popcouncil.org), or Effie Chipeta, (echipeta@medcol.mwn).

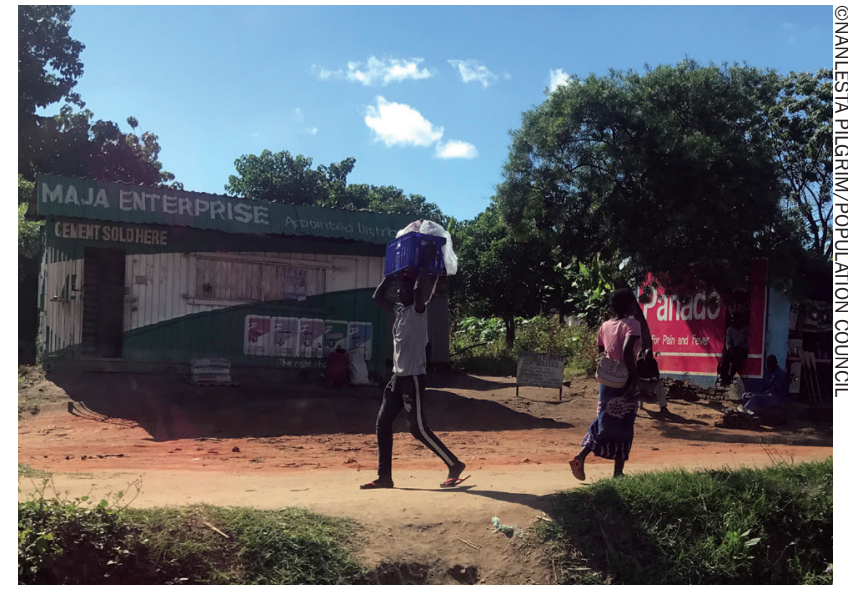

This study will build needed evidence to inform decision-making about effective programming to reduce HIV risk among AGYW and their male partners.

interventions that go beyond the health sector, addressing the structural drivers that fuel AGYW's HIV risk.

Project SOAR is conducting implementation science research to build needed evidence to inform decision-making about effective programming to reduce HIV risk among AGYW and their male partners. Specifically, SOAR is:

1. Examining approaches to identify the most at-risk girls, particularly those out of school, and enroll and retain them in DREAMS programming;

2. Assessing HIV-risk and health-seeking behaviors before and after AGYW's engagement in DREAMS interventions;

3. Exploring how to reach AGYW's male sexual partners and increase their uptake of HIV services; and

4. Assessing facilitators and barriers to HIV service uptake by men living with HIV. 
Learnings from this study, and similar ones in Kenya and Zambia, will be valuable for the U.S. President's Plan for Emergency AIDS Relief (PEPFAR) program implementation in Malawi and the region, as efforts around HIV prevention among AGYW expand. They also will be relevant globally, given the increased attention by the international AIDS community to fostering an AIDS-free generation.

\section{Our Research}

The research is being carried out in Machinga and Zomba Districts where DREAMS interventions are taking place. These districts have high HIV prevalence, large numbers of orphans and vulnerable children, high prevalence of early sexual initiation, large treatment gaps, high rates of childbearing during the teen years, and high school drop-out rates among girls. The research uses secondary data analyses and quantitative and qualitative methods.

Finding the most at-risk girls: SOAR is analyzing previously collected quantitative cohort data from the Population Council's Malawi Schooling and Adolescent study to identify the individual, partner, and community characteristics associated with being infected with HIV and/or herpes simplex virus. These characteristics will be compared to those of DREAMS participants to determine whether DREAMS implementers are engaging the most at-risk AGYW, and if not, how they might better reach them.

Assessing outcomes: SOAR is following a cohort of DREAMS out-of-school beneficiaries to determine the effect of DREAMS programming in reducing their HIV-related risk behaviors. Qualitative data collected from AGYW and program staff will help us better understand these changes as well as the degree to which the DREAMS comprehensive services were delivered as designed.

Reaching male partners: SOAR is carrying out community mapping exercises with AGYW and community leaders to identify locations where
AGYW meet male partners. Male partners recruited at identified locations will participate in in-depth interviews to determine how to reach them in order to increase their use of HIV services. The data collected will determine the types of men with whom AGYW engage in sexual partnerships, where to find them, and what they want from HIV services, including what has worked so far and what has not.

Improving male service uptake: SOAR is conducting qualitative interviews with men who are living with HIV to determine barriers and facilitators to HIV service use.

\section{Research Utilization}

The DREAMS partnership involves many stakeholders, including from U.S. Agency for International Development, the DREAMS PEPFAR team in Malawi, Office of the Global AIDS Coordinator Technical Advisor for DREAMS Malawi, the Malawi Ministry of Health, the Malawi National AIDS Commission, and the DREAMS implementing partners. As a first step, SOAR will identify a smaller group of key stakeholders from the above organizations/institutions to serve as the Research Advisory Committee (RAC). Individuals on the RAC will both provide guidance to the study and serve as a primary audience for the study findings.

SOAR will engage the RAC at key points during the study's implementation so that members are continually appraised of progress and have opportunities to provide input. Key points of strategic engagement with the RAC will include data collection to address any challenges that emerge, data interpretation to identify the study's implications and recommendations, and results communication to encourage uptake of the findings by decisionmakers.

${ }^{1}$ Ministry of Health. 2016. "Summary sheet preliminary
findings: The Malawi population-based HIV impact
assessment." New York: Columbia University. https://www.
hiv.health.gov.mw/images/Documents/MALAWIFactsheet.pdf
Project SOAR is a five-year (September 2014-September 2019) cooperative agreement funded by the President's Emergency Plan for AIDS Relief and the U. S. Agency for International Development (Agreement No. AIDOAA-A-14-00060). SOAR is able to accept funding from all USAID accounts.

Population Council leads the Project SOAR consortium in collaboration with Avenir Health, Elizabeth Glaser Pediatric AIDS Foundation, the Johns Hopkins University, Palladium, and The University of North Carolina at Chapel Hill.
Project SOAR/Population Council

4301 Connecticut Avenue, NW, Suite 280

Washington, DC 20008

Tel: +1 2022379400

e-mail: ProjectSOAR@popcouncil.org

popcouncil.org/ProjectSOAR

(c)Population Council, May 2017 